These results agree with a recent observation that the proportion of teenage pregnancies aborted is much higher in the prosperous south eastern regions of England than in the northern regions. ${ }^{6}$

As in the English study, the differences within Tayside did not seem to be related to the availability of abortions on the National Health Service. Geographically most of the deprived areas are closer to the two hospitals where most abortions were carried out than are the affluent areas. Moreover, the rate of teenage abortions in Tayside is higher than the Scottish average, ${ }^{4}$ and facilities are generally considered to meet the demand.

It is not known how many of the girls recorded as resident in the deprived areas had moved there from more affluent areas to have their babies as a result of changed relationships with parents and boyfriends arising from their pregnancy. A recent analysis of previous addresses on the community health index, however, suggests that this was probably not an important factor in their high rate of live births. Out of a sample of 31 girls living in the most deprived areas (deprivation category 7) when they gave birth in 1992, 23 were at the same address before the start of their pregnancies, and those who moved there during pregnancy all came from the same or other deprived areas (categories 5 and 6). Neither is it possible to say whether a higher proportion of the girls resident in the deprived areas who become pregnant were in stable relationships than of those girls in the affluent areas because of the decline in recent years in the popularity among teenagers of an official marriage ceremony; in 1980 some 9029 teenage girls got married in Scotland compared with only 2285 in $1990 .^{7}$ In my analysis of girls who had had two pregnancies as teenagers the high proportion of single girls who kept both of their babies suggests that many were in fact in stable relationships despite the designation.
The current unreliability of the term "single" when applied to marital status in young people emphasises the need for an additional option to be included in all record systems to distinguish between people who are genuinely single and those in a stable relationship but not officially married.

For girls who are genuinely single having a child, whether planned or unplanned, may be more socially acceptable in the deprived areas than in the more affluent areas. This raises the worrying question of whether girls in the most affluent areas are placed under undue social and parental pressure to terminate their pregnancies, possibly against their real wishes.

In terms of absolute numbers of girls involved (rather than rates) the problem of unwanted teenage pregnancies as measured by abortions is clearly as much one of affluent areas as deprived areas.

The need to reduce the risk of unwanted pregnancy has assumed greater urgency in recent years because of the concomitant risk of contracting HIV (against which the morning after pill offers no defence), and thus the problem of preventing unwanted conceptions in the under $16 \mathrm{~s}$, in teenagers as a whole, and indeed in all age groups has to be urgently addressed.

\section{Secretary of State for Health. The health of the nation; a strategy for health in England. London: HMSO, 1992. (CM 1986.) \\ 2 OPCS. Birth Statistics 1990, 19. London: HMSO, 1992 \\ 3 The Scottish Office. Scotland's health, a challenge to us all. Edinburgh: HMSO, 1992. \\ 4 Information and Statistics Division, Common Services Agency. Scottish health statistics. Edinburgh: ISD Publications, 1991. \\ 5 Carstairs V, Morris R. Deprivation and health in Scotland. Aberdeen: Aberdeen University Press, 1991. \\ 6 Wilson SH, Brown TP, Richards RG. Teenage conceptions and contraception in the English Regions. Fournal of Public Health Medicine 1992;14:17-25. \\ 7 General Register Office for Scotland. Annual repon of the registrar general for Scotland 1990. No 136. Edinburgh: HMSO, 1991. \\ $8 \mathrm{Smith} \mathrm{T}$. The relative effects of sex and deprivation on the risk of premature} death. Foumal of Rublic Health Medicine 1992;14:402-7.
Microalbuminuria Collaborative Study Group Members of the study group are listed at the end of the paper.

Correspondence to: Dr Jeannie Yip, Unit for Metabolic Medicine, United Medical and Dental Schools, Guy's Hospital, London SE1 9RT.

BMY 1993;306:1235-9

\title{
Risk factors for development of microalbuminuria in insulin dependent diabetic patients: a cohort study
}

\author{
Microalbuminuria Collaborative Study Group, United Kingdom
}

\section{Abstract}

Objective-To investigate the risk factors for the development of persistent microalbuminuria in insulin dependent diabetic patients.

Design-Four year follow up of a cohort of diabetic patients.

Setting-Outpatient departments of teaching and district general hospitals in England.

Subjects-148 non-microalbuminuric, nonhypertensive insulin dependent diabetic patients.

Main outcome measures-Urinary albumin excretion rate and arterial blood pressure.

Results-137 patients completed four year follow up, of whom 11 developed persistent microalbuminuria (albumin excretion rate $\geqslant 30 \mu \mathrm{g} / \mathrm{min}$ on at least two consecutive occasions), giving a cumulative frequency of $8 \% .103$ remained normoalbuminuric and 23 exhibited intermittent microalbuminuria. At baseline the persistent microalbuminuric group had had significantly raised blood pressure (mean 137.9 (SD 14.9)/82.3 (7.6) v 123.5 (13.2)/72.8 (9.1) mm Hg), glycated haemoglobin concentration $10.4 \%(2.0 \%) v$ $8-9 \%(2 \cdot 0 \%)$, and albumin excretion rate (median (interquartile range) $17.5(13 \cdot 0-22 \cdot 3) v 0.8$ (3.7-6.7) $\mu g / m i n)(p<0.05$ for all) compared with the normoalbuminuric group. Blood pressure and glycated haemoglobin remained significantly higher in the persistent microalbuminuric group throughout the study $(p<0.05)$. Multiple regression analysis showed initial albumin excretion rate, blood pressure, and smoking to be significant determinants of persistent microalbuminuria $(p<0.02)$.

Conclusion-In insulin dependent diabetic patients with poor glucose control, which may initially increase albumin excretion rate, an early rise of arterial pressure and smoking are implicated in the development of persistent microalbuminuria.

\section{Introduction}

The reasons for the susceptibility to nephropathy of a subset of insulin dependent diabetic patients remain elusive. The two clinical hallmarks of overt diabetic kidney disease are persistent proteinuria and arterial hypertension.

Microalbuminuria, an early marker of progression to overt renal disease in insulin dependent diabetes, ${ }^{1-4}$ has been found to be associated with poor glycaemic control $^{5}$ and raised arterial pressure. ${ }^{245}$ Changes in these variables seem to occur in parallel in microalbuminuric patients, ${ }^{6}$ but the cause-effect relation between them is unclear and the factors responsible for 
the transition from normoalbuminuria to microalbuminuria are unknown. Answering these questions would help in the understanding of the pathogenesis of diabetic renal disease. We therefore investigated the factors associated with the progression from nonmicroalbuminuria to persistent microalbuminuria and explored the roles of metabolic control and arterial pressure in an observation study of a cohort of nonmicroalbuminuric insulin dependent diabetic patients.

\section{Patients and methods}

All insulin dependent diabetic patients attending nine outpatient diabetic clinics between 1984 and 1986 were invited to participate in a screening programme for urinary albumin excretion rate. ${ }^{7}$ They had to fulfil the following selection criteria: age $16-60$ years, onset of diabetes before age 39 years, duration of disease less than 35 years, no albuminuria by dipstick test, sitting blood pressure below $160 / 95 \mathrm{~mm} \mathrm{Hg}$, and no antihypertensive treatment or clinical evidence of cardiac or renal disease. Absence of microalbuminuria was defined as an overnight albumin excretion rate $<30$ $\mu \mathrm{g} / \mathrm{min}$ since only excretion rates above this level have been found to be consistently predictive of progression to clinical proteinuria ${ }^{1389}$ and to be associated with significantly more severe renal lesions. ${ }^{10}$ Whether levels of albumin excretion rates between the upper limit of the normal distribution in non-diabetic patients $(12 \mu \mathrm{g} / \mathrm{min}$, calculated as the mean $+2 \mathrm{SD})$ and $30 \mu \mathrm{g} / \mathrm{min}$ predict overt nephropathy remains unclear. ${ }^{1-489}$ One report based on short term urine collection under water diuresis suggested that albumin excretion rates greater than $15 \mu \mathrm{g} / \mathrm{min}$ may also be predictive. $^{4}$

The screening phase of this study has been described elsewhere. ${ }^{7}$ In brief, of 1885 eligible insulin dependent diabetic patients, 1500 (group 1) had an urinary albumin concentration $<15 \mu \mathrm{g} / \mathrm{ml}$ or albumin:creatinine ratio $<3.5 \mathrm{mg} / \mathrm{mmol}$, or both, in an early morning urine specimen. These values are highly specific for normal urinary albumin excretion rate. ${ }^{11}$ The remaining 385 patients (group 2) had values equal to or above these figures. These patients were further characterised by two timed overnight urine collections within two months. Two hundred and fifty five patients provided timed overnight samples, of whom 202 were found to have an urinary albumin excretion rate consistently below $30 \mu \mathrm{g} / \mathrm{min}$. We also invited a random sample of 144 patients from group 1 to provide a timed overnight urine collection ${ }^{7} ; 83$ complied and all had an albumin excretion rate below $30 \mu \mathrm{g} / \mathrm{min}$. The median (interquartile range) excretion rate was 6.6 $(4 \cdot 0-12 \cdot 6) \mu \mathrm{g} / \mathrm{ml}$ for patients in group 2 and 4.9 (3.7$7.5) \mu \mathrm{g} / \mathrm{ml}$ for group $1(p<0.01)$. Although significantly different, these two values were well within the accepted normal range in all prospective studies of albumin excretion rates ${ }^{1-41} 13$ and the two groups were therefore combined for the follow up study.

All 285 patients were asked to take part in a longitudinal study. Fifty three patients from group 1 and 95 from group 2 ( 79 men and 69 women) gave informed consent to the follow up protocol, which was approved by the ethics committees of the participating centres. These 148 patients had similar clinical characteristics to those who declined to participate (age (30.3 (SD12.0) $v 32.2(11.7)$ years)), duration of diabetes $14.4(10.9) v 15.3(9.4)$ years, and screening urinary albumin:creatinine ratio $(2.57$ (3.6) $v 2.62$ $(2 \cdot 3) \mathrm{mg} / \mathrm{mmol}$ ).

Patients were all receiving conventional insulin treatment, taking a diabetic diet, and seen every six months by research registrars who worked to a standardised manual of operations. At each visit sitting blood pressure (phase I and V) was recorded twice to the nearest $2 \mathrm{~mm} \mathrm{Hg}$. Readings were taken five minutes apart, after 10 minutes' rest, in the dominant arm with a random zero sphygmomanometer. The average of the two readings was used for the analyses. Mean blood pressure was derived from diastolic pressure plus one third pulse pressure. Blood was taken to measure glycated haemoglobin and plasma creatinine concentrations. Albumin excretion rate was measured in a sterile timed overnight urine collection. Funduscopy was done through dilated pupils with a standardised method of assessment used by the World Health Organisation multinational study. ${ }^{4}$ Retinopathy was classified as none, minimal, medium, or severe. ${ }^{15}$ Patients were classified as smokers if they currently smoked or had smoked over five cigarettes a day in the past or non-smokers if they had never smoked.

Arterial hypertension was defined as sitting blood pressure $>160 / 95 \mathrm{~mm} \mathrm{Hg}$ measured on two occasions over two weeks. The primary end point of the study was the development of persistent microalbuminuria defined as albumin excretion rate $>30 \mu \mathrm{g} / \mathrm{min}$ on two consecutive measurements six months apart, the time of onset being taken as the second of the two measurements.

\section{LABORATORY MEASUREMENTS}

Urinary albumin concentration was measured in a central laboratory at Guy's Hospital by a radioimmunoassay with interassay and intra-assay variations of $10 \cdot 2 \%$ and $5 \cdot 1 \%$ respectively. ${ }^{7}$ Glycated haemoglobin (Corning electroendosmosis) and plasma creatinine concentrations Jaffe reaction rate method, Hitachi autoanalyser, Boehringer, Lewes, were measured at three of the participating centres: Guy's Hospital, Poole General Hospital, and the Royal Victoria Infirmary, Newcastle upon Tyne. The results obtained at the other two hospitals were regularly checked at the central laboratory at Guy's Hospital to ensure comparability.

\section{STATISTICAL ANALYSES}

The analysis was based on the results from the four year follow up visit for all patients. Analysis of variance, unpaired $t$ tests, and Fisher's exact test were used as appropriate. Analysis of variance for repeated measurements was used to compare differences between groups throughout the study period. The rate of change of albumin excretion rate was estimated from the slope of the linear regression analysis of albumin excretion rate against time. Correlation analysis between albumin excretion rate and a selection of variables was assessed by Pearson's regression analysis. Multiple stepwise logistic regression analyses were used to examine the importance of several initial parameters on the outcome variable final albumin excretion rate. Parameters were treated as continuous or categorical variables. Urinary albumin excretion rates were logarithmically transformed before statistical analysis. A p value below 0.05 was considered significant. Results are expressed as mean (SD) or median (interquartile range). The SAS statistical package was used for all data processing. ${ }^{16}$

\section{Results}

Eleven patients (three men, eight women) dropped out of the study after a follow up period of 12 to 42 months. One was severely brain damaged in a road traffic accident; six moved out of the area, and four were unwilling to continue with the study. Of the remaining 137 patients, 11 (five men, six women) developed persistent microalbuminuria; eight were from group 2 and three from group 1 . One hundred and three patients (60 men, 43 women) remained 
normoalbuminuric and 23 ( $11 \mathrm{men}, 12$ women) had an albumin excretion rate $>30 \mu \mathrm{g} / \mathrm{min}$ on one or more non-consecutive occasions (intermittent microalbuminuria). Five of the 11 patients who developed persistent microalbuminuria went through a phase of intermittent microalbuminuria. The cumulative frequency for the development of microalbuminuria was $8 \%$ calculated by a standard life table method. ${ }^{17}$

Patients who remained normoalbuminuric or developed persistent or intermittent microalbuminuria were of similar age and body mass index but the duration of diabetes tended to be shorter in patients who remained normoalbuminuric (table I). Baseline arterial pressure, albumin excretion rate, and glycated haemoglobin concentration were all significantly higher in the patients who developed persistent microalbuminuria compared with those in patients who remained normoalbuminuric (table II). These differences remained after matching for duration of diabetes. A significantly higher proportion of smokers progressed to persistent microalbuminuria $14 \%(9 / 66)$ than non-smokers $3 \%(2 / 71)(p=0.03)$.

Diabetic patients who developed intermittent microalbuminuria had blood pressure and albumin excretion rate values which were intermediate between those in the other two groups and worse glycaemic control than the persistent normoalbuminuric group (table II). Mean (SD) daily insulin doses at baseline were similar in all three groups (persistent normoalbuminuria $50.7(18.3)$, persistent microalbuminuria $46 \cdot 9(16 \cdot 2)$, intermittent microalbuminuria $54 \cdot 4(21 \cdot 1)$ U/day).

Mean albumin excretion rate, blood pressure, and glycated haemoglobin concentration remained significantly higher in the persistent microalbuminuric group compared with the persistent normoalbuminuric group throughout the study $(p<0.05)$ (figure). The average rate of change of albumin excretion rates was $36 \%$ a year in the persistent microalbuminuric group, $8 \%$ a year in the persistent normoalbuminuric group $(p<0.05)$, and $17 \%$ a year in the intermittent microalbuminuric group. One $(9 \%)$ microalbuminuric patient, four $(4 \%)$ normoalbuminuric patients, and one $(4 \%)$ intermittent microalbuminuric patient were started on antihypertensive drugs; this difference was not significant.

Of all the baseline parameters, only glycated haemoglobin concentration and mean blood pressure were significantly though weakly correlated $(r=0 \cdot 19$, $\mathrm{p}<0.05)$. In univariate logistic regression analysis initial albumin excretion rate $(\beta=4.22, p<0.001)$, mean blood pressure $(\beta=0 \cdot 17, p<0.001)$, glycated

TABLE I-Baseline demographic features of insulin dependent diabetic patients who remained persistently normoalbuminuric, progressed to persistent microalbuminuria, or developed intermittent microalbuminuria

\begin{tabular}{lccc}
\hline & $\begin{array}{c}\text { Persistent } \\
\text { normoalbuminuric } \\
(\mathrm{n}=103)\end{array}$ & $\begin{array}{c}\text { Persistent } \\
\text { microalbuminuria } \\
(\mathrm{n}=11)\end{array}$ & $\begin{array}{c}\text { Intermittent } \\
\text { microalbuminuria } \\
(\mathrm{n}=23)\end{array}$ \\
\hline Sex (M/F) & $60 / 43$ & $5 / 6$ & $11 / 12$ \\
Median (range) age (years) & $32(18-57)$ & $33(25-56)$ & $32(18-55)$ \\
Median (range) duration (years) & $12(1-33)$ & $19(13-29)$ & $14(2-33)$ \\
Mean (SD) body mass index $\left(\mathrm{kg} / \mathrm{m}^{2}\right)$ & $24 \cdot 8(4 \cdot 1)$ & $24 \cdot 5(3 \cdot 0)$ & $25 \cdot 6(3 \cdot 8)$ \\
\hline
\end{tabular}

TABLE II-Baseline systolic blood pressure, diastolic blood pressure, albumin excretion rate, and glycated haemoglobin concentration in insulin dependent diabetic patients who remained persistently normoalbuminuric, progressed to persistent microalbuminuria, or developed intermittent microalbuminuria

\begin{tabular}{|c|c|c|c|}
\hline & $\begin{array}{c}\text { Persistent } \\
\text { normoalbuminuric } \\
(\mathrm{n}=103)\end{array}$ & $\begin{array}{l}\text { Persistent } \\
\text { microalbuminuria } \\
(n=11)\end{array}$ & $\begin{array}{c}\text { Intermittent } \\
\text { microalbuminuria } \\
(\mathrm{n}=23)\end{array}$ \\
\hline $\begin{array}{l}\text { Mean (SD systolic pressure ( } \mathrm{mm} \mathrm{Hg} \text { ) } \\
\text { Mean (SD) diastolic pressure (mm Hg) } \\
\text { Median (interquartile range) albumin }\end{array}$ & $\begin{array}{l}123 \cdot 5(13 \cdot 2) \\
72 \cdot 8(9 \cdot 1)\end{array}$ & $\begin{array}{c}137 \cdot 9(14 \cdot 9)^{\star} \\
82 \cdot 3(7 \cdot 6)^{\star}\end{array}$ & $\begin{array}{l}125 \cdot 9(10 \cdot 1) \ddagger \\
75 \cdot 4(6 \cdot 7)\end{array}$ \\
\hline $\begin{array}{l}\text { excretion ( } \mu \mathrm{m} / \mathrm{min}) \\
\text { Mean (SD) glycated haemoglobin (\%) }\end{array}$ & $\begin{array}{l}4 \cdot 8(3 \cdot 7-6 \cdot 7) \\
8.9(2 \cdot 0)\end{array}$ & $\begin{array}{c}17 \cdot 5(13 \cdot 0-22 \cdot 3)^{\star} \\
10 \cdot 4(2 \cdot 0)^{\star}\end{array}$ & $\begin{array}{c}13 \cdot 5(4 \cdot 4-21 \cdot 8) \dagger \\
10 \cdot 0(2 \cdot 4) \dagger\end{array}$ \\
\hline
\end{tabular}

* $p<0.05$ compared with normoalbuminuric group.

tp $<0.05$ compared with normoalbuminuric group.

$\neq p<0.05$ compard with persistent microalbuminuria group.

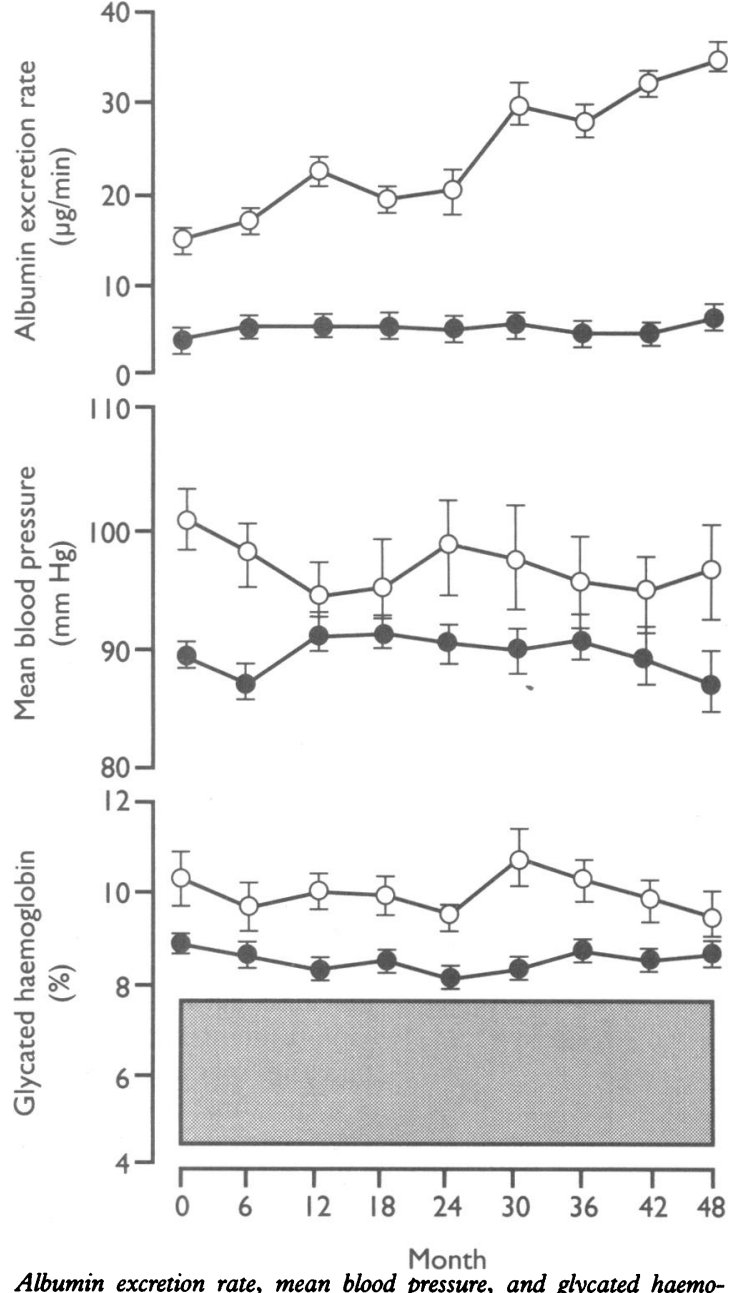

globin concentration in insulin dependent diabetic patients who progressed to persistent microalbuminuria (O) and in those who remained normoalbuminuric (O) throughout the four year study period. The shaded area in the bottom panel represents the normal range of glycated haemoglobin concentration. Results are in mean (SD) and geometric mean (SD) for albumin excretion rate.

TABLE III-Stepwise multiple logistic regression analysis relating initial albumin excretion rate, mean blood pressure, glycated haemoglobin concentration, smoking, age, and duration of diabetes to development of microalbuminuria in 137 insulin dependent diabetic patients

\begin{tabular}{lccc}
\hline Independent variables & $\begin{array}{c}\boldsymbol{\beta} \\
\text { Coefficient }\end{array}$ & $\begin{array}{c}\text { Standard } \\
\text { error }\end{array}$ & $\begin{array}{c}\mathrm{p} \\
\text { Value }\end{array}$ \\
\hline Albumin excretion rate & $4 \cdot 10$ & 1.64 & 0.012 \\
Mean blood pressure & 0.23 & 0.08 & 0.003 \\
Smoking & 2.13 & 1.81 & 0.049 \\
\hline
\end{tabular}

Glycated haemoglobin $(p=0 \cdot 18)$, age $(p=0.98)$, and duration of diabetes $(p=0.25)$ were not significant and eliminated from the model.

haemoglobin $(\beta=0.36, p<0.05)$, and history of smoking $(\beta=1 \cdot 7, p=0.03)$ were all significantly related to the development of persistent microalbuminuria. To allow for the interaction between these variables initial albumin excretion rate, mean blood pressure, glycated haemoglobin concentration, history of smoking, and age and duration of diabetes were entered as independent variables in a backward, stepwise, multiple logistic regression analysis with microalbuminuria as the dependent outcome variable. Initial albumin excretion rate, mean blood pressure, and smoking emerged as significant determinants of microalbuminuria (table III).

Initial blood pressure remained a significant predictor of microalbuminuria whether systolic blood pressure $(\beta=0.13, p<0.003)$ or diastolic blood pressure $(\beta=0.18, p<0.01)$ was entered into the model. When albumin excretion rate was used as a continuous outcome variable, initial albumin excretion rate and mean blood pressure persisted as significant determi- 
nants in both univariate $(\beta=0.26, p<0.001$ and $\beta=$ $0.01, p<0.01$, respectively) and multiple regression analysis $(\beta=0.27, p=0.001$ and $\beta=0.009, p=0.02$ respectively).

The proportion of patients with severe retinopathy at baseline was significantly higher in the persistent microalbuminuric (three, 27\%) and intermittent microalbuminuric (six, 26\%) groups than in the persistent normoalbuminuric group (five, $5 \% ; p<0.05$ Fisher's exact test). These differences remained at the four year follow up (four, 37\%; five, 24\%; and eight, $8 \%$, respectively). Mean (SD) plasma creatinine concentration remained within the normal range in all patients throughout the study without significant differences between the three groups (persistent normoalbuminuria $84.5 \quad(8 \cdot 1) \mu \mathrm{mol} / /$, persistent microalbuminuria $82.7(3.6) \mu \mathrm{mol} / /$, and intermittent microalbuminuria $89 \cdot 2(4 \cdot 8) \mu \mathrm{mol} / \mathrm{l})$.

To compare our results with those of other workers who used albumin excretion rate $>20 \mu \mathrm{g} / \mathrm{min}$ as the definition of microalbuminuria ${ }^{12}{ }^{13}$ we also analysed our data using an excretion rate $>20 \mu \mathrm{g} / \mathrm{min}$ as the cut off point for microalbuminuria. At entry into the study 131 patients had an albumin excretion rate $<20 \mu \mathrm{g} /$ min. Over the observation period 92 remained below $20 \mu \mathrm{g} / \mathrm{min}, 17$ progressed to persistent microalbuminuria (as defined by two consecutive measurements of albumin excretion rate $>20 \mu \mathrm{g} / \mathrm{min}$ six months apart) giving a cumulative frequency of $13 \%$, and 22 had an intermittent pattern (one or more non-consecutive measurements $>20 \mu \mathrm{g} / \mathrm{min}$ ).

The three groups of patients were comparable for age (normoalbuminuria $30.9(19.8)$, persistent microalbuminuria $31 \cdot 7(4 \cdot 1)$, intermittent microalbuminuria 34.7 (13.1) years), duration of diabetes (14.5 (22.1), 14.2 (4.5), 16.2 (9.4) years) and body mass index $\left(25 \cdot 2(7 \cdot 7), 24 \cdot 4(2 \cdot 1), 25 \cdot 6(3 \cdot 8) \mathrm{kg} / \mathrm{m}^{2}\right)$ at entry. Notably patients who became persistently microalbuminuric had a significantly higher initial mean blood pressure $(100.3(7.8)$ v $89.6(9.6) \mathrm{mm} \mathrm{Hg}$, $\mathrm{p}<0.05)$ and initial albumin excretion rate $(15.7(13.0$ 17.5) $v 4.6(3.5-6 \cdot 7) \mu \mathrm{g} / \mathrm{min}, \mathrm{p}<0.05)$ than those who remained normoalbuminuric. Glycated haemoglobin concentration was slightly higher at baseline in the patients who developed microalbuminuria but the difference was not significant $(9.9 \%(1.6 \%) v 8.9 \%$ $(1.9 \%), p=0.06)$. In a stepwise multiple logistic regression analysis initial albumin excretion rate $(\beta=3.97$, $\mathrm{p}<0.001)$ and mean blood pressure $(\beta=0.18$, $\mathrm{p}=0.002$ ) emerged as significant determinants of microalbuminuria.

\section{Discussion}

In this four year prospective study of nonmicroalbuminuria insulin dependent diabetic patients, 11 out of 137 patients developed persistent microalbuminuria, a cumulative frequency of $8 \%$. About $40 \%$ of patients failed to complete the two initial screening procedures, though the compliers and noncompliers were demographically and clinically similar. Patients with the poorest attendance at clinics are at greater risk of nephropathy ${ }^{18}$ and thus our study could have underestimated the progression to persistent microalbuminuria. However, this is unlikely since the observed four year cumulative frequency of $8 \%$ corresponds closely with the yearly incidence of diabetic nephropathy in insulin dependent diabetic patients, which varies from $1 \%$ to $3 \% .{ }^{19}$

The average increase in albumin excretion rate of $36 \%$ a year in the patients who became microalbuminuric was similar to that reported by Feldt-Rasmussen et al for microalbuminuric patients progressing to overt proteinuria. ${ }^{\circ}$
BLOOD PRESSURE AND BLOOD GLUCOSE CONTROL

The patients who progressed to microalbuminuria had had raised blood pressure and albumin excretion rate before the persistent microalbuminuria became established. Moreover blood glucose control remained consistently worse and blood pressure consistently higher in the group of progressors throughout the follow up. Blood pressure and smoking were independent determinants of microalbuminuria, thus clarifying the prognostic importance of these variables which has been suggested in cross sectional studies. ${ }^{2450}$ The predictive power of glycaemia seemed to be weaker. The contribution of poor metabolic control to the development of diabetic renal complications is supported by several studies. ${ }^{561221}$

Patients who developed intermittent microalbuminuria also had significantly worse glycaemic control at baseline than those who remained normoalbuminuric. Their blood pressure was intermediate between that of the other two groups. These findings suggest that worse blood glucose control may lead to the initial rise in albuminuria and that raised blood pressure, as seen in the patients who became persistently microalbuminuric, may increase the speed of deterioration of albumin excretion rate. Smoking, a recognised risk factor for vascular damage, ${ }^{22}$ would further aggravate the process.

It could be argued that our use of a cut off point for albumin excretion rate of $30 \mu \mathrm{g} / \mathrm{min}$ included patients who, by other authors' definition, had already developed microalbuminuria and had slightly raised arterial pressure. This is, however, unlikely to be the case because when data were analysed with an albumin excretion rate $<20 \mu \mathrm{g} / \mathrm{min}$ as the cut off point the same pattern of findings emerged. Our results strongly suggest that raised albumin excretion rate and blood pressure are parallel and concomitant manifestations of a common disease process.

\section{COMPARISONS WITH OTHER STUDIES}

Our results are similar to those of Mathiesen $e t a l^{12}$ in that both studies showed that the group of progessors had had a raised albumin excretion rate and worse glycaemic control at baseline. These results disagree with the findings of Rudberg et al, who reported no difference in these variables in a group of insulin dependent diabetic adolescents. ${ }^{23}$

Our results differ from those of Mathiesen $e t$ al in a crucial aspect. They reported that a rise in blood pressure occurred over two years after the onset of persistent microalbuminuria and concluded that increased arterial pressure is secondary to and a consequence of microalbuminuria. The demographic features and the percentage of patients developing microalbuminuria were similar in the two studies. However, our study was prospective and included regular, standardised measurements of defined variables whereas the Danish study assessed albumin excretion rate more often than blood pressure and glycated haemoglobin. Moreover, in the Danish study blood pressure was recorded by different observers in an outpatient clinic with a standard mercury sphygmomanometer and readings were taken to the nearest $5 \mathrm{~mm} \mathrm{Hg}$, potentially introducing important interassay and intraobserver variations and loss of precision. Small but important changes in blood pressure could easily have been missed in this way. Our data indicate that if there is a time lag between the beginning of the rise in blood pressure and albumin excretion rate it must occur early, when these variables are increasing within their normal ranges; the precise timing of such changes can be resolved only by observing patients with shorter duration of diabetes. It is clear, however, that the albumin excretion rate does not have to rise 
into the microalbuminuria range before it induces a rise in blood pressure.

Insulin dependent diabetic patients who developed persistent or intermittent microalbuminuria were significantly more likely to have retinal lesions at baseline than those who remained normoalbuminuric. This difference was maintained at follow up and has also been shown by other workers. ${ }^{24}$

In conclusion our study shows that raised albumin excretion rate and blood pressure are concomitant phenomena in insulin dependent diabetic patients progressing to microalbuminuria. A background of poor blood glucose control compounded with a rise in blood pressure and, to a lesser extent, a history of smoking are crucial determinants of this process.

Members of the study group were Colin F Close, Andrea Collins, Angela Goodwin, Caron Hill, R John Jarrett, Sharon L Jones, Harry Keen, Graham S Scott, GianCarlo Viberti, Hita Vora, and Jeannie Yip, Guy's Hospital, London; Anna Grenfell, Michael J Sampson, and Peter J Watkins, King's College Hospital, London; Carol Fishwick, Wendy Gatling and Ronald D Hill, Poole General Hospital; and Sally M Marshall, University of Newcastle upon Tyne.

This work was supported by a grant from the Department of Health and by Guy's Hospital Special Trustees. We thank the following colleagues for allowing us to study their patients: Dr N Essex, Mayday University Hospital, Croydon; Dr G Jackson, Lewisham Hospital, London; Dr C Lowry and Professor P Sonksen, St Thomas's Hospital, London; Dr P Marsden, Greenwich District Hospital, London; Dr P Stephenson, Queen Elizabeth Hospital, Gateshead; Dr E T Young, Ashington General Hospital; and Dr J Yudkin, Whittington Hospital, London.

1 Viberti GC, Hill RD, Jarrett RJ, Argyropoulos A, Mahmud U, Keen H. Microalbuminuria as a predictor of clinical nephropathy in insulindependent diabetes mellitus. Lancet 1982;i:1430-2.

2 Mathiesen ER, Øxenboll B, Johansen K, Svendsen PA, Deckert T. Incipient nephropathy in type 1 (insulin-dependent) diabetes. Diabetologia 1984;26: 406-10.

3 Parving HH, Øxenboll B, Svendsen PA, Christiansen JS, Andersen AR. Early detection of patients at risk of developing diabetic nephropathy: a longitudinal study of urinary albumin excretion. Acta Endocrinol 1982;100:500-5.

4 Mogensen CE, Christensen CK. Predicting diabetic nephropathy in insulindependent patients. N Engl $\mathcal{G}$ Med 1984;311:89-93.
5 Wiseman MJ, Viberti GC, Mackintosh D, Jarrett RJ, Keen H. Glycaemia, arterial pressure and microalbuminuria in type 1 (insulin-dependent) diabetes mellitus. Diabetologia 1984;26:402-5.

6 Feldt-Rasmussen B, Mathiesen ER, Deckert T. Effect of two years of strict metabolic control on progression of incipient nephropathy in insulindependent diabetes. Lancet 1986;ii:1300-4.

7 Microalbuminuria Collaborative Study Group, UK. Microalbuminuria in type 1 (insulin-dependent) diabetic patients: prevalence and clinical characteristics. Diabetes Care 1992;4:495-501.

8 Messent JWC, Elliott TG, Hill RD, Jarrett RJ, Keen H, Viberti GC. Prognostic significance of microalbuminuria in insulin-dependent diabetes mellitus-a 23 year follow-up study. Kidney Int 1992;41:836-9.

9 Feldt-Rasmussen B, Mathiesen ER, Jensen T, Lauritzen T, Deckert T. Effect of improved metabolic control on loss of kidney function in type 1 (insulindependent) diabetic patients: an update of the Steno studies. Diabetologia dependent) diabetic

10 Chavers BM, Bilous RW, Ellis EN, Steffes MW, Mauer SM. Glomerular lesions and urinary albumin excretion in type 1 diabetes without overt proteinuria. N Engl f Med 1989;320:966-70.

11 Gatling W, Knight C, Hill RD. Screening for early diabetic nephropathy: which sample to detect microalbuminuria? Diabetic Med 1985;2:451-5.

12 Mathiesen ER, Renn B, Jensen T, Storm B, Deckert T. The relationship between blood pressure and urinary albumin excretion in the developmen of microalbuminuria. Diabetes 1990;39:245-9.

13 Mogensen CE, Chachati A, Close CF, Deckert T, Honnel E, et al. Microalbuminuria: an early marker of renal involvement in diabetes. Uraemia Invest 1986;9:85-95.

14 World Health Organisation Multinational Study of Vascular Disease in Diabetes. Prevalence of small vessel and large vessel disease in diabetic patients from 14 centres. Diabetologia 1985;28:615-40.

15 Jarrett RJ, Keen H, Grabauskas V. The WHO multinational study of vascular disease in diabetes: general description. Diabetes Care 1979;2:175-86.

16 SAS users' guide: statistics: version 5. Cary, North Carolina: SAS Institute, 1985.

17 Armitage P, Berry G. Statistical methods in medical research. 2nd ed. Oxford: Blackwells, 1987:408-14

18 Krolewski AS, Warram JH, Christlieb AR, Busick EJ, Khan CR. The changing natural history of neophropathy in type 1 diabetes. $\mathrm{Am} \mathrm{f}$ Med 1985;78:785-94

19 Andersen AR, Christiansen JS, Andersen JK, Kreiner S, Deckert T. Diabetic nephropathy in type 1 (insulin-dependent) diabetes: an epidemiological study Diabetologia 1983;25:496-501.

20 Chase HP, Garg SK, Marshall G, Begg CL, Harris F, Jackson WE, et al. Cigarette smoking increases the risk of albuminuria among subjects with type 1 diabetes. JAMA 1991;265:614-7.

21 The Kroc Collaborative Study Group. Blood glucose control and evoluation of diabetic retinopathy and albuminuria. $N$ Engl f Med 1984;6:2365-9.

22 Reid DD, Hamilton PJS, McCartney P, Rose G, Jarrett RJ, Keen H. Smoking and other risk factors for coronary heart disease in British civil servants. Lancet 1976;ii:979-84.

23 Rudberg S, Persson B, Dahlquist G. Increased glomerular filtration rate as a predictor of diabetic nephropathy -an 8-year prospective study. Kidney In 1992;41:822-8.

24 Vigstrup J, Mogensen CE. Prolifrative diabetic retinopathy: at risk patients identified by early detection of microalbuminuria. Acta Ophthalmol 1985;63: $530-43$

(Accepted 2 March 1993)
University of Sheffield

Department of Paediatrics,

Section of Paediatric

Haematology, Children's

Hospital, Sheffield

S102TH

Helena Davies, Leukaemia

Research Fund clinical fellow

John Lilleyman, head of

section

University of Sheffield

Department of Medicine,

Section of Pharmacology

and Therapeutics, Royal

Hallamshire Hospital,

Sheffield S10 2JF

Lynne Lennard, research

scientist

Correspondence to:

Dr Lilleyman.

BMF 1993;306:1239-40

\section{Variable mercaptopurine metabolism in children with leukaemia: a problem of non-compliance?}

\author{
Helena A Davies, Lynne Lennard, \\ John S Lilleyman
}

The successful treatment of childhood lymphoblastic leukaemia depends in part on extended oral medication with mercaptopurine. The most important group of metabolites of mercaptopurine, 6-thioguanine nucleotides, accumulate slowly in red cells to reach a steady state concentration in nearly all children after one to four weeks.' The concentration of these metabolites correlates with cytotoxicity and can be used to assess the adequacy of treatment in a way broadly analogous to the measurement of glycated haemoglobin in the control of diabetes.

Some interpatient variability in the concentration of 6-thioguanine nucleotides is undoubtedly due to differences in the way patients metabolise mercaptopurine, ${ }^{2}$ but we have seen intrapatient variability that needs an alternative explanation. Scant consideration has been given to the question of compliance, ${ }^{34}$ so we undertook a preliminary study to see if children with acute lymphoblastic leukaemia always take their mercaptopurine tablets.

\section{Patients, methods, and results}

We recruited 35 consecutive children with acute lymphoblastic leukaemia who were treated in Sheffield or Nottingham, were in their first remission, and had been receiving similar maintenance chemotherapy that included continuous treatment with mercaptopurine at a standard daily dose of $75 \mathrm{mg} / \mathrm{m}^{2}$ for at least nine months. The children were seen at least fortnightly, and their drug doses were reduced if they developed neutropenia or thrombocytopenia. On the basis of whether they had ever taken the standard dose of mercaptopurine for four or more weeks continuously without developing cytopenias, we designated 22 of the children as tolerant of the treatment and 13 as nontolerant.

After the tolerant children had completed at least four weeks of treatment with the standard dose of mercaptopurine (when the concentration of 6thioguanine nucleotides in red cells should have been reaching a steady value) we took blood samples to measure the concentration of these metabolites. The blood was taken at the time of routine venepuncture, and the methods of measuring the metabolites have been described elsewhere. ${ }^{5}$ We also assessed the compliance of all 35 children with their treatment by means of a structured interview. 